\title{
Examination of Single-phase Partial Discharge Monitoring Device for Partial Discharge Diagnosis on Three-phase GIS
}

\author{
Umar Khayam $^{1}$, Shinya Ohtsuka ${ }^{1}$, Masayuki Hikita ${ }^{1}$, \\ Nobuko Otaka ${ }^{2}$, Takakazu Matsuyama ${ }^{2}$, \\ Yoshiki Takehara ${ }^{3}$ \\ ${ }^{1}$ Kyushu Institute of Technology, Department of Electrical and Electronic Engineering, \\ 1-1 Sensui-cho, Tobata-ku, Kitakyushu, 804-8550 Japan \\ ${ }^{2}$ Japan AE Power Systems Corporation, 8-3, Nishi-Shimbashi 3-chome, \\ Minato-ku, Tokyo, 105-0003 Japan \\ ${ }^{3}$ Hitachi Engineering and Services Co.,Ltd., 1-1, Kokubo-cho 1-chome, \\ Hitachi-shi, Ibaraki, 316-8501 Japan \\ umar@hv.ee.itb.ac.id
}

\begin{abstract}
This paper deals with partial discharge induced electromagnetic wave investigation on three-phase GIS using UHF method and the application of single-phase partial discharge monitoring device (PDM) on three-phase gas insulated switchgear (GIS). The PD source is a protrusion at different positions on the conductor or on the tank. Frequency and phase spectrums of EMW signals emitted by PD at different positions were observed. The most dominant frequency of EMW signal in three-phase GIS was estimated from the cutoff frequency (fc) formula for single-phase GIS. The main difference in frequency spectrum between three-phase and single-phase GIS was found to be the shift of fc to the lower value for three-phase GIS. The presence of three conductors can be considered as increase in an equivalent radius of three-phase conductor by a factor of 1.6 for this GIS model. The phase of PD phase pattern in the phase spectrum depended on the particle position. The PD sources on three-phase GIS were diagnosed by single-phase PDM device. The success rate of diagnosis results is low. The error is predicted to be caused by error in phase recognition of PD pattern. The change in phase shift setting of PD phase pattern in PDM device based on the phase angle of applied voltage at maximum electric field gave better diagnosis results.
\end{abstract}

Index Terms: three-phase, GIS, PDM device, phase shift setting, success rate

\section{Introduction}

In general, three-phase equipment differs from single-phase equipment in two aspects: configuration and applied voltage. These differences result in different phenomena of electric field and partial discharge (PD) between single-phase and three-phase equipment. The special characteristics of electric field and PD in three-phase equipment, such as three-phase power cable and three-phase gas insulated switchgear (GIS) model have been reported recently [1-6]. The development of PD diagnostic device for three-phase equipment and application of singlephase PD monitoring device (PDM) on three-phase GIS should consider these special characteristics.

Nowadays single-phase PD monitoring device has been being developed and applied on single-phase equipment. PD data base of PDM device is obtained from single-phase equipment. Because the application of three-phase GIS in electric power network has been increasing, it is very important to develop PDM device for three-phase GIS. It is convenient and saving in cost if single-phase PDM device can be used and applied for PD diagnosis in three-phase GIS. Therefore, the examination of application of single-phase PDM device on three-phase GIS is needed.

Received: September 1,2010. Accepted: September 25, 2010 
This research examines the possibility of application of single-phase PD monitoring device (PDM) for PD diagnosis on three-phase GIS model. The PD source is a protrusion on the conductor or a protrusion on the tank. Electromagnetic wave (EMW) emitted by PD in threephase GIS was detected by UHF sensor. The success rate of PDM was examined for a particle attached on different positions. The error in diagnosis result was analyzed. In addition, the effect of particle and sensor position on the success rate of PDM were examined and analyzed.

\section{Experimental Setup}

Three-phase GIS model, the experimental setup, UHF sensors position and notation, are shown in Figure 1 and Figure 2. Particle position and notation are described in Figure 3. The particles which simulate protrusion were put on S45 to produce PD in the phase-phase region and S270 and E270 to produce PD in the phase-tank region. The internal UHF sensors, UHF $\mathrm{A}, \mathrm{B}, \mathrm{C}$, and D are installed inside the three-phase GIS for measurement of EMW signals excited by PD. UHF A, B, and D are installed in the smaller tank while UHF C is installed in the bigger tank. The sensitivity of UHF sensors is below $0.3 \mathrm{pC}$.

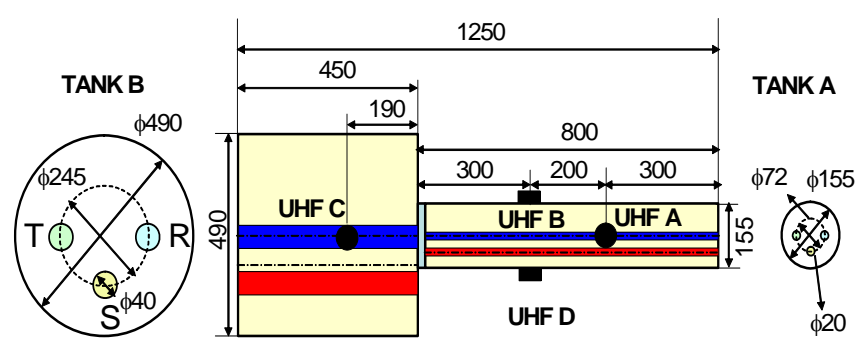

Figure 1. Layout and dimension of three-phase GIS model

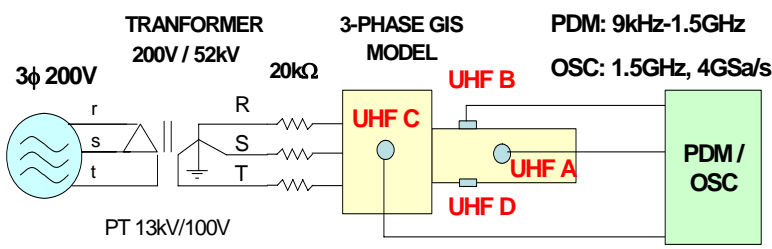

Figure 2. Experimental setup

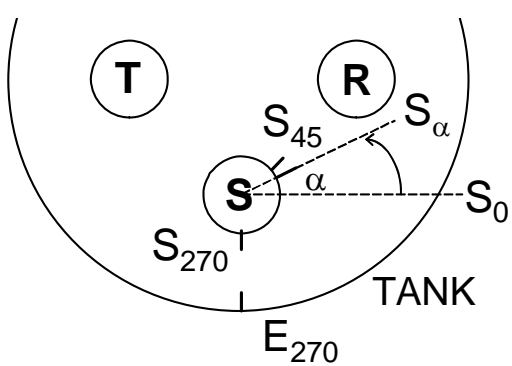

Figure 3. Particle position and notation 


\section{Partial Discharge Monitoring Device}

Figure 4 and Figure 5 show the diagram of PDM device examined in this research and its masking system respectively. EMW emitted by PD was detected with UHF sensors and diagnosed with single phase PDM device by expert diagnostic software [7,8]. The PDM contains spectrum analyzer with frequency band $9 \mathrm{kHz}-1.5 \mathrm{GHz}$. A measured signal is compared with the reference waveforms stored in a personal computer. The external noises are sometimes detected by UHF sensor and disturb diagnosis of PD. Therefore, this system has masking function to ignore the external noises such as mobile, aircraft and machine noise etc.

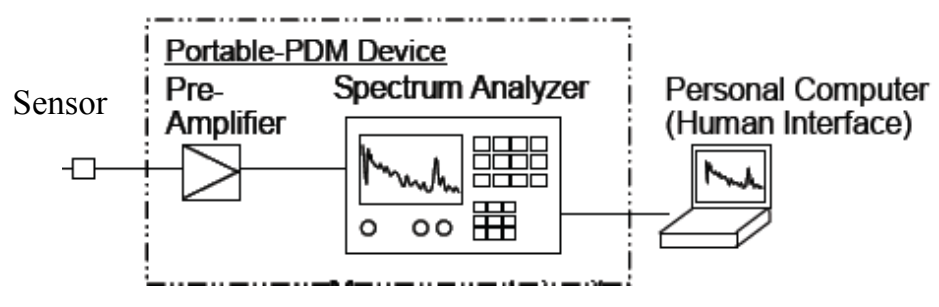

Figure 4. PDM Device

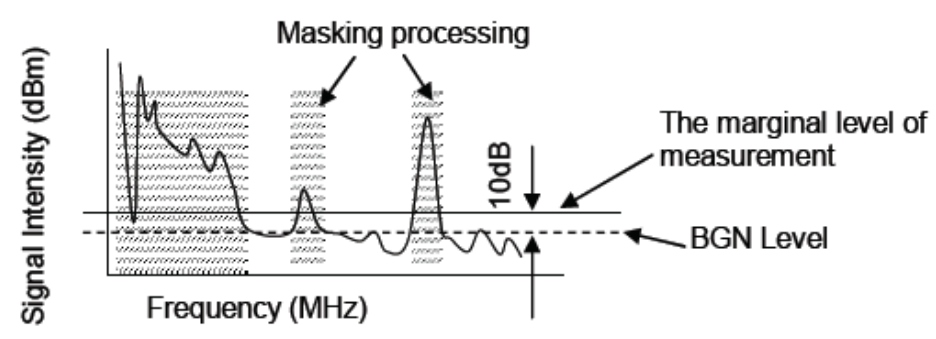

Figure 5. Masking system of PDM device

The reference waveform is obtained from various PD sources in single-phase GIS: protrusion, particle on the spacer, floating electrode, spacer crack, and external noise. Figure 6 shows the typical of phase spectrum pattern in the data base. At the first stage, actual PD and external noise signals are classified by means of phase patterns. Then, through the neural network, one reference waveform is selected to identify the PD cause. Figure 7 shows procedure of diagnosis by neural network having special branch structures.

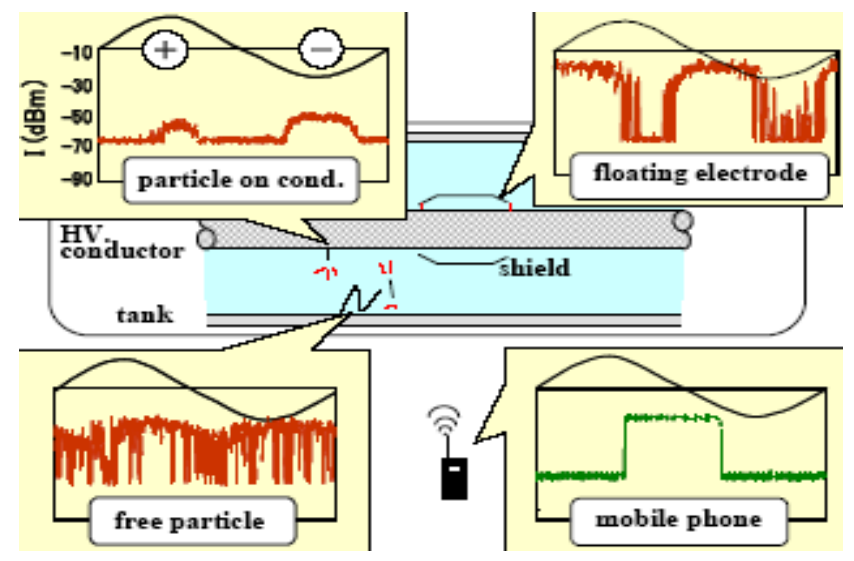

Figure 6. PD phase patterns of mobile phone and various PD sources in PDM data base 
PDM provides synchronization facility so that the phase of PDM power source and the phase of experiment power source ( $\mathrm{S}$ phase voltage in this experiment) can be synchronized. Because the phase pattern is used for the diagnosis, the phase standard is necessary. S phase was measured as a standard phase in this experiment. The phase angle was changed and examined afterwards. The change of phase angle to the base phase was assumed to be $\beta$.

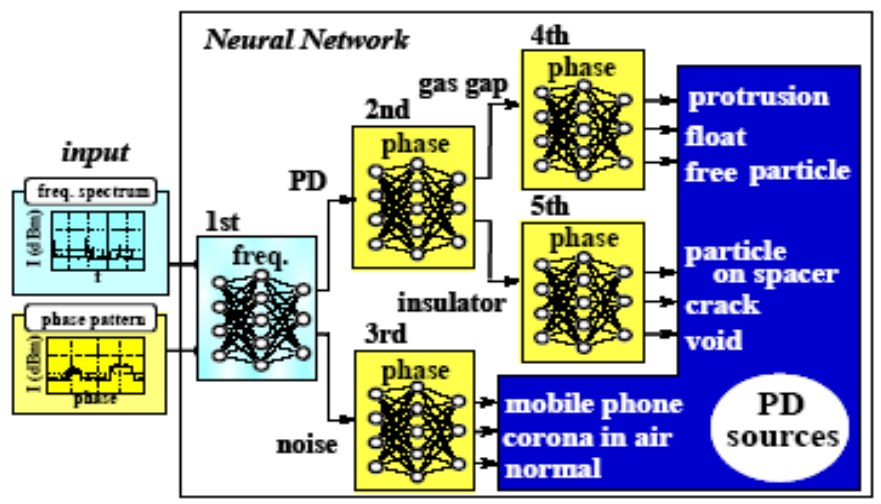

Figure 7. Procedure of diagnosis by neural network having special branch structures

\section{Experimental Results}

A. Frequency and Phase Spectrum of PD induced EMW in three-phase GIS
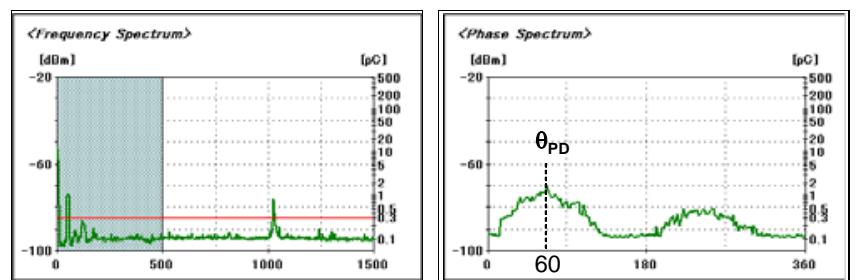

a. S270
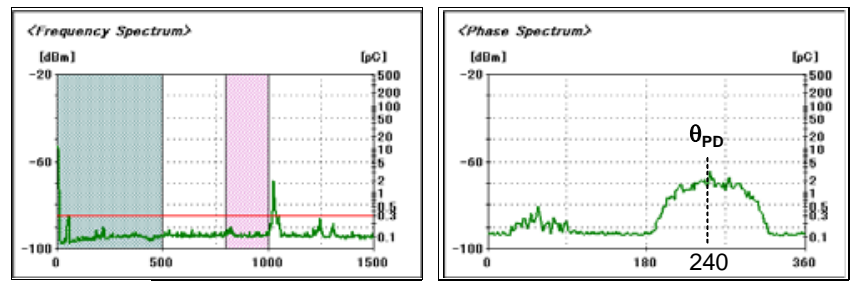

b. E270
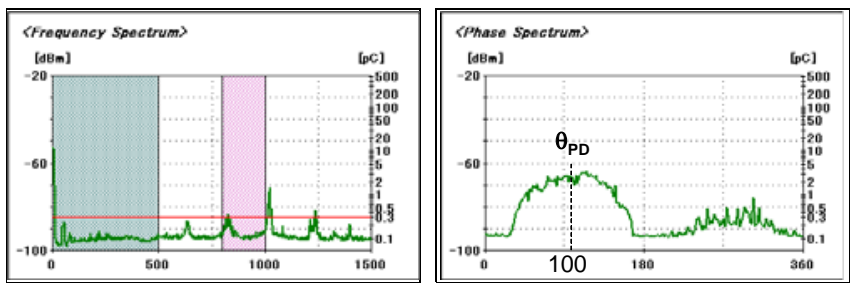

c. S45

Figure 8. Frequency and phase spectrum of EMW emitted by PD for S270, E270, and S45 particle detected with UHF $A\left(p=0.3 \mathrm{MPa}, \mathrm{V}_{\mathrm{a}}=16 \mathrm{kV}\right)$. Frequency is in $\mathrm{MHz}$, phase is in degree, and $\mathrm{EMW}$ intensity in $\mathrm{dBm}$ and $\mathrm{pC}$. 
Figure 8 shows experimental results of frequency and phase spectrums of EMW emitted by PD measured with UHF A for S270, E270, and S45 particles at gas pressure p=0.3 MPa and applied voltage $\mathrm{Va}=16 \mathrm{kV}$. The most dominant frequency $\mathrm{f}_{\mathrm{d}}$ in frequency spectrum appears at 1018, 1025, and $1027 \mathrm{MHz}$ for S45, S270, and E270, respectively. PDM identifies automatically this frequency as the center frequency. The phase spectrum is taken at this frequency. As shown in Figure 8 the phase spectrum consists of two mountains in a cycle. $\theta_{\mathrm{PD}}$ is defined as phase of single-phase voltage or $\mathrm{S}$ applied voltage at the middle point of larger mountain in PD phase spectrum measured in the experiment. $\theta_{\mathrm{PD}}$ is shown as dashed lines in Figure 4. $\theta_{\mathrm{PD}}=60^{\circ}, 240^{\circ}$, and $100^{\circ}$ for S270, E270, and S45 particles, respectively. $\theta_{\mathrm{PD}}$ depended on the particle position.

\section{B. PDM Diagnosis Result}

Figure 9 summarizes diagnosis results of PDM based on EMW signals measured with UHF A when a particle at S45, E270, or S270 was attached in GIS model. Diagnosis is success if a protrusion is detected as protrusion. Success rate $\rho$ means presentation of success diagnosis results to all diagnosis results.

It is found that success rate $\rho$ is $69 \%, 6 \%$, and $1 \%$ for S45, E270, and S270 particles, respectively. The success rate $\rho$ of diagnosis mainly for particles on S270 and E270 is very low.

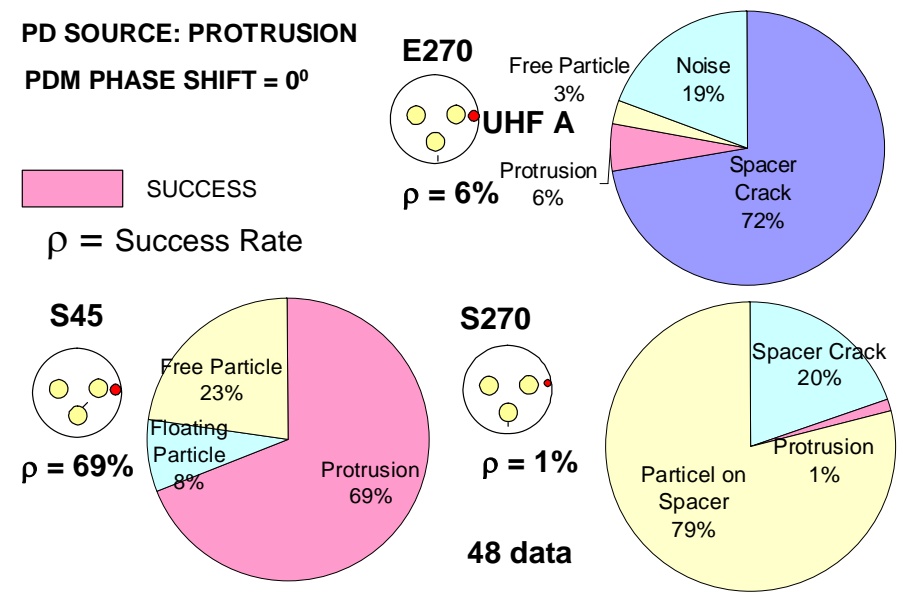

Figure 9. PD diagnosis results by PDM device. Success rate $\rho$ is defined as presentation of success diagnosis results to all diagnosis results.

\section{Discussion}

A. Frequency Spectrum of PD induced EMW in three-phase GIS

Frequency spectrum of EMW emitted by PD describes EMW propagation characteristics inside GIS. It is well known that there are three categories of EMW which can be excited and propagate in coaxial cylindrical structures like GIS, namely, TEM, TE, and TM modes. The electromagnetic waves in TEmn and TMmn modes can not exist at frequencies below the cutoff frequency $\left(f_{c}\right)$. The characterization and identification of each mode are very useful to understand PD phenomena in GIS. Generally, the peaks in the frequency spectrum excited by PD describe each mode and the resonant characteristics, namely cutoff frequency $\left(f_{c}\right)$ and resonant frequency $\left(\mathrm{f}_{\mathrm{r}}\right)$.

Let's analyze the most dominant frequency of the frequency spectrum shown in Figure 8 by estimation of cutoff frequency of EMW signals in three-phase GIS. In the calculation of cutoff frequency, the models as shown in Figure 10 are examined. The model A approximates 
$\mathrm{f}_{\mathrm{c}}$ considering only tank A, while the model $\mathrm{B}$ approximates $\mathrm{f}_{\mathrm{c}}$ considering only tank B. For each model, there are three types calculation: I, II, III.

Model I considers the actual three-phase GIS structure. Model II approximates $\mathrm{f}_{\mathrm{c}}$ using single phase formula of $f_{c}$, with the single phase conductor radius equal to one of three-phase conductor radius. Model III is same with model II, with the single-phase conductor radius equal to the radius of the circle connecting three-phase conductors. The special formula of cutoff frequency $f_{c}$ for three-phase GIS has not been derived yet. Therefore, the calculation of cutoff frequency $f_{c}$ of three-phase GIS will be estimated with the formula for single-phase GIS.

The cutoff frequency $f_{c}$ for single-phase GIS, with the outer radius of the conductor $r_{o}$ and the inner radius of the tank be $r_{i}$, can be estimated by these formulas $[8,9]$.

$$
\mathrm{f}_{\mathrm{c}}=\mathrm{c} / \lambda_{\mathrm{c}}
$$

$\mathrm{TM}_{\mathrm{mn}}$ wave:

$$
\lambda_{\mathrm{c}}=2\left(\mathrm{r}_{\mathrm{o}}-\mathrm{r}_{\mathrm{i}}\right) / \mathrm{n} \quad \text { for }(\mathrm{m}=0,1, \ldots, \mathrm{n}=1,2, \ldots)
$$

$\mathrm{TE}_{\mathrm{mn}}$ wave:

$$
\begin{aligned}
& \lambda_{\mathrm{c}}=\pi\left(\mathrm{r}_{\mathrm{o}}+\mathrm{r}_{\mathrm{i}}\right) / \mathrm{m} \quad \text { for }(\mathrm{m}=1,2, \ldots, \mathrm{n}=1) \\
& \lambda_{\mathrm{c}}=2\left(\mathrm{r}_{\mathrm{o}}-\mathrm{r}_{\mathrm{i}}\right) /(\mathrm{n}-1) \text { for }(\mathrm{m}=1,2, \ldots, \mathrm{n}=2,3, \ldots)
\end{aligned}
$$

Since the model is three-phase lumped buses and not in coaxial cylindrical shape, this analytical equation can not be applied strictly speaking. However, because the equivalent $r_{i}$ is a small value with respect to $r_{0}$, that much large error may not occur even by assuming a similar form with single-phase bus.

Table 1 contains the calculation results of $\mathrm{TE}_{11}$ mode for $\mathrm{A}_{\mathrm{II}}, \mathrm{A}_{\mathrm{III}}, \mathrm{B}_{\mathrm{II}}$, and $\mathrm{B}_{\mathrm{III}}$ model and the comparison with the frequency dominant of frequency spectrum resulted from the measurement.

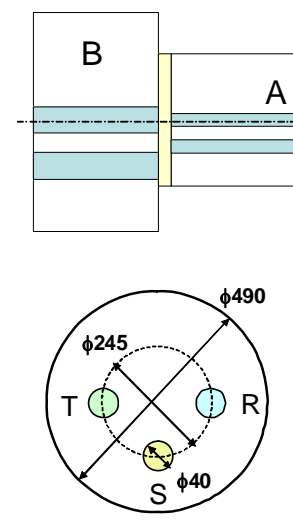

$B_{1}$

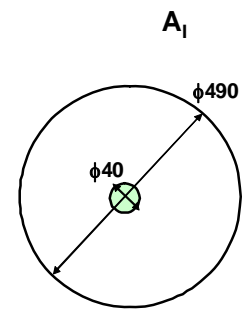

$\mathbf{B}_{\| 1}$
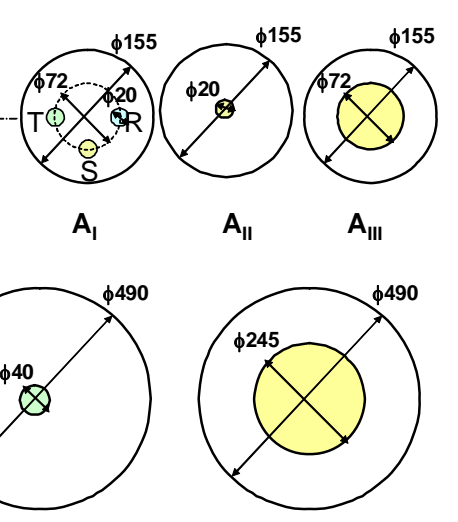

$B_{\text {III }}$

Figure 10. Model for calculation of cutoff frequencies 
Table 1 Cutoff frequency $f_{c}$ (calculation results) and dominant frequency $\mathrm{f}_{\mathrm{d}}$ (measurement results)

\begin{tabular}{|c|c|c|c|c|c|c|c|}
\hline METHODE & \multicolumn{3}{|c|}{ CALCULATION } & \multicolumn{3}{c|}{ MEASUREMENT } \\
\hline MODEL & $A_{\| I}$ & $A_{\| I I}$ & $B_{\| I}$ & $B_{\| I}$ & S45 & E270 & S270 \\
\hline fC(MHz) & 1,092 & 842 & 361 & 265 & 1,018 & 1,027 & 1,025 \\
\hline
\end{tabular}

It appears from Table 1 that only $\mathrm{f}_{\mathrm{c}} \mathrm{TE}_{11}$ of $\mathrm{A}_{\mathrm{II}}$ model be close to $\mathrm{f}_{\mathrm{d}}$. As mentioned previously, model $A_{\text {II }}$ only considers the small tank and approximates $f_{c}$ using single phase formula of $f_{c}$, with the single phase conductor radius equal to one of three-phase conductor radius. This result indicates that formula of $f_{c}$ for single-phase GIS may be used for calculation of $f_{c}$ on three-phase GIS. This result also indicates that the most dominant frequency $f_{d}$ of the frequency spectrum emitted by PD can be attributed to the first cutoff frequency of EMW signal in three-phase GIS. The cutoff frequency $\mathrm{f}_{\mathrm{c}}$ are $1018 \mathrm{MHz}, 1027 \mathrm{MHz}$, and $1025 \mathrm{MHz}$ for S45, E270, and S270 particles, respectively.

However, there is a little difference in the cut off frequency of EMW signals between the calculation $\left(\mathrm{fc}_{\mathrm{AI}}=1092 \mathrm{MHz}\right)$ and the measurement results $\left(\mathrm{fc}_{3 \phi} \cong 1025 \mathrm{MHz}\right)$. The difference can be attributed to the difference between single-phase GIS and three-phase GIS because the calculation is based on the single phase GIS while the measurement is resulted from threephase GIS. The $\mathrm{fc}$ in three phase GIS is lower than fc in single-phase GIS. The decrease of $\mathrm{fc}$ may be considered as the increase of radius $\mathbf{r}_{\text {ieq }}$ of the single-phase equivalent conductor as the replacement of three conductors in three-phase GIS [2]. The equivalent radius is predicted larger than the radius of single phase conductor $\left(r_{\text {ieq }}>r_{i}\right)$ based on equation 3 . The calculation results are summarized in Table 2 .

Table 2

The calculation results of equivalent radius of three-phase conductor

\begin{tabular}{|l|l|l|}
\hline \multicolumn{1}{|c|}{ Parameter } & \multicolumn{1}{c|}{$1 \Phi$} & \multicolumn{1}{c|}{$3 \Phi$} \\
\hline $\mathrm{f}_{\mathrm{c}}(\mathrm{MHz})$ & 1092 & 1025 \\
\hline$\lambda_{\mathrm{c}}(\mathrm{m})$ & 0.27 & 0.29 \\
\hline $\mathrm{r}_{\mathrm{o}}(\mathrm{mm})$ & 77.5 & 77.5 \\
\hline $\mathrm{r}_{\mathrm{i}}(\mathrm{mm})$ & 10 & 16 \\
\hline
\end{tabular}

It is found that equivalent radius of three-phase GIS is 1.6 times larger than the radius of single-phase conductor $\left(\mathrm{r}_{\mathrm{ieq}}=1.6 \mathrm{r}_{\mathrm{i}}\right)$. Therefore, the presence of three conductors can be considered as the increase in radius equivalent $r_{\text {ieq }}$ of three-phase conductor while it reduces the cutoff frequency $f_{c}$.

\section{B. Phase Spectrum of PD induced EMW in three-phase GIS}

Experimental results show that $\theta_{\mathrm{PD}}$ in the phase spectrum of PD induced EMW signal depended on the particle positions. These results are explained by the electric field at the particle tip inside three-phase GIS.

Figure 11 shows periodic change of absolute electric field on the tips of S45 and E270 particles at $1 \mathrm{kV}$ applied voltage during one cycle of $\mathrm{S}$ applied voltage. $\theta_{\mathrm{s} \text { max }}$ is defined as the phase angle of $\mathrm{S}$ applied voltage when the electric field on the particle tip achieves maximum value. Maximum electric field at the particle tip differs depending on the particle position. Maximum electric field occurs at $\theta \mathrm{s}=90$ and $\theta \mathrm{s}=270$ for $\mathrm{E} 270$ and $\theta \mathrm{s}=113$ and $\theta \mathrm{s}=293$ for $\mathrm{S} 45$. Phases where maximum electric field at the particle tip occurs differed depending on the particle position. Because PD pulse sequence of protrusion appears around $\theta_{\mathrm{s} \max }[10]$, or $\theta_{\mathrm{PD}}=$ 
$\theta_{\mathrm{s} \max }, \theta_{\mathrm{PD}}$ also depends on the particle position in three-phase GIS. It explains the experimental results: i.e. $\theta_{\mathrm{PD}}$ depended on the particle position.

In single-phase GIS PD caused by protrusion always occurs at the peak of applied voltage [10] and independent of particle position, while in three-phase GIS there is dependence of phase where PD pulse occurs on particle position [11].

However, $\theta_{\mathrm{PD}}$ as shown in Figure 8 is not same with $\theta_{\mathrm{s} \max }$ shown in Figure 11 . The difference is caused by the phase difference between PDM power source and three-phase power source. These results should be considered in the practical application, mainly in the application of single-phase PDM to 3 phase GIS.

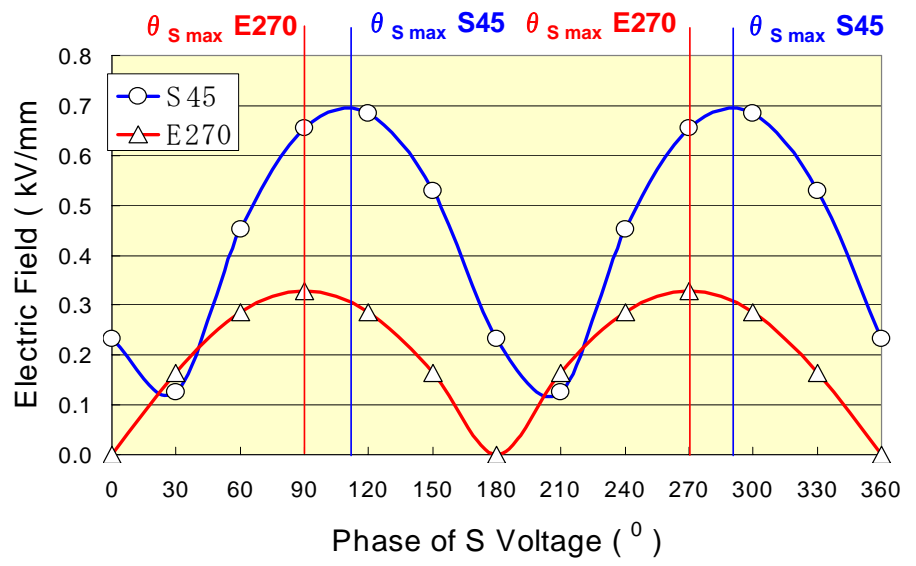

Figure 11. Periodic change of electric field on the particle tips

\section{Analysis of PDM Diagnosis Results}

Experimental results show that application of single-phase PDM device gave low success rate of diagnosis results. Note that success rate $\rho$ means presentation of success diagnosis results to all diagnosis results.

As mentioned previously, PDM device identifies PD sources based on the spectrum of PD induced EMW signal. Therefore, the low in success rate may be caused by error in recognition of PD pattern. Note that the reference waveform in PDM is obtained from data base of singlephase GIS whereas there are differences in frequency and phase spectrums of EMW signal between single-phase and three-phase GIS as explained in $A$ and $B$.

PDM takes PD phase pattern at the frequency where EMW signal has maximum signal to noise ratio. PDM detects the change or the shift in dominant frequency. Therefore, PDM is independent from the difference in cut-off frequency between single-phase and three-phase GIS. Thus, the low in success rate of PDM may be caused by the error in recognition of PD pattern. In single-phase GIS PD caused by protrusion always occurs at the peak of applied voltage [9] while in three-phase GIS there is dependence of phase where PD pulse occurs on particle position [10].

Figure 12 shows calculated electric field on the particle tip of S45, S270 and E270 particles at $1 \mathrm{kV}$ during one cycle of $\mathrm{S}$ phase voltage. $\theta_{\mathrm{s} \text { max }}$ is defined as the phase angle of S applied voltage when the electric field on the particle tip achieves maximum value. PD pulse sequence of protrusion appears around $\theta_{\mathrm{s} \max }$ [9]. $\theta_{\mathrm{s} \text { max }}$ is shown as solid line in Figure 12. $\Delta$ is defined as the phase differences between $\theta_{\mathrm{s} \max }$ and $\theta_{\mathrm{PD}}$. It is found that in a cycle of $\mathrm{S}$ applied voltage $\theta_{\mathrm{s}}$ max $=113^{\circ}$ and $\theta_{\mathrm{s} \text { max }}=293^{\circ}$ for S45, while $\theta_{\mathrm{s} \max }=90^{\circ}$ and $\theta_{\mathrm{s} \max }=270^{\circ}$ for S270 and E270. It is estimated that $\Delta=150^{\circ}$ or $\Delta=-210^{\circ}$ for E270 and S270, while $\Delta=-193^{\circ}$ or $\Delta=167^{\circ}$ for S45. Phase of PD pattern in PDM should be translated as $\beta=\Delta$ to achieve correct phase spectrum. 


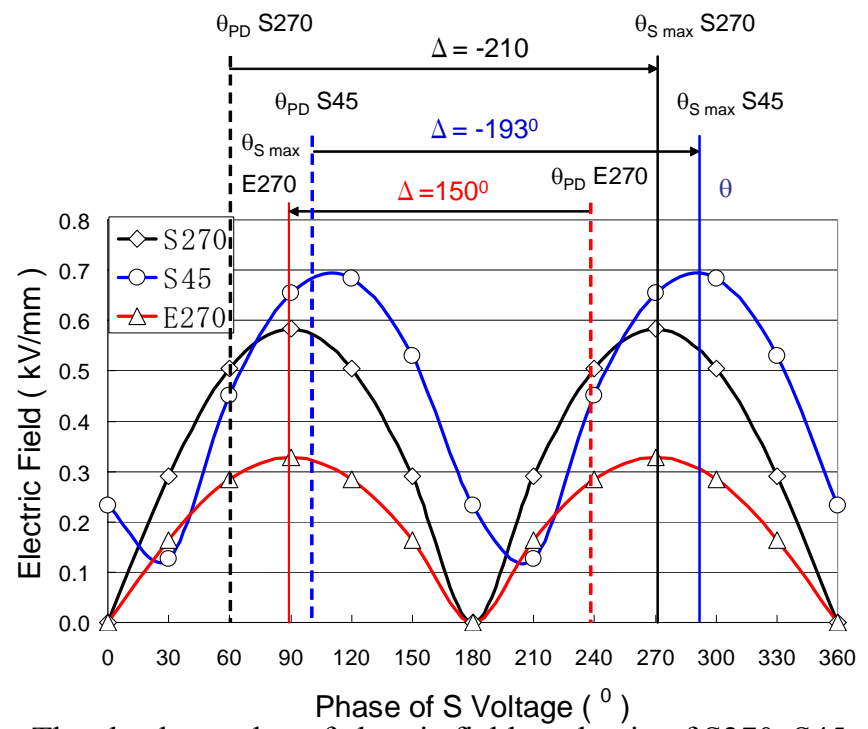

Figure 12. The absolute value of electric field on the tip of S270, S45, and E270 particles

\section{Effect of Phase Shift Setting on PDM Diagnosis Results}

PDM provides phase shift $\beta$ setting facility so that phase of PD pattern may be shifted [9]. Based on the electric field calculation above, the best phase shift setting of PDM are estimated to be $\beta=-193^{\circ}$ or $\beta=167^{\circ}$ for $\mathrm{S} 45$, and $\beta=-193^{\circ}$ or $\beta=167^{\circ}$ and $\beta=-210^{\circ}$ or $\beta=150^{\circ}$ for E270, and S270 particles.

Figure 13 shows the effect of phase shift setting on success rate for S45, S270, and E270 particles UHF A, for $\beta=0$ and $\beta=\Delta$. Note that success rate $\rho$ means presentation of success diagnosis results to all diagnosis results.

After the phase shift setting was changed to the proper value, the success rate increased to almost $100 \%$. This result indicated that the change in phase shift $\beta$ setting in PDM device based on the phase angle of applied voltage at maximum electric field on the particle tip gave better diagnosis results.

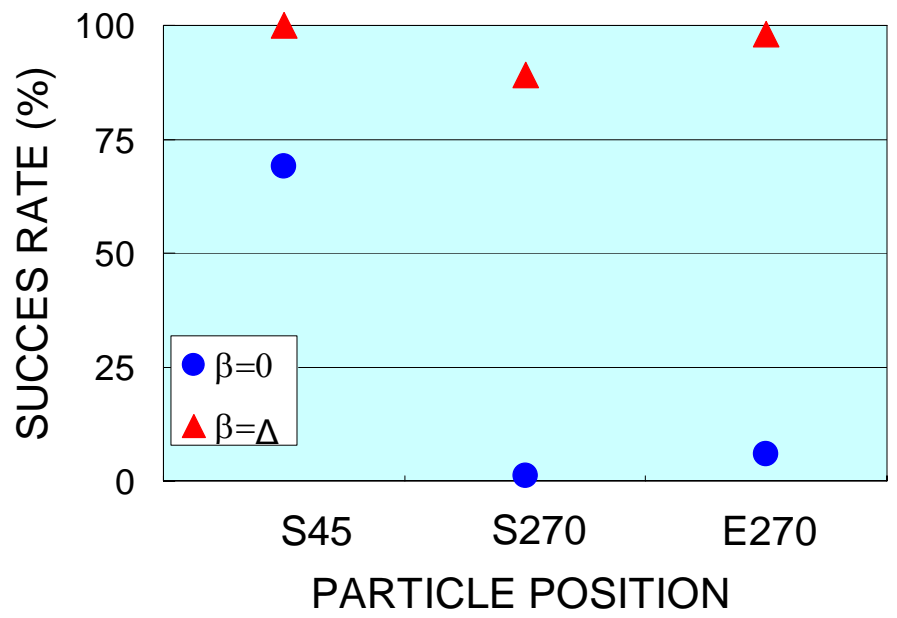

Figure 13. Effect of phase shift setting on success rate for S45, S270, and E270 particles (UHF A, $3 \mathrm{~atm}(\beta=0), 2 \mathrm{~atm}(\beta=\Delta)$ ). Success rate $\rho$ means presentation of success diagnosis results to all diagnosis results. 


\section{Conclusion}

We investigated partial discharge in three-phase GIS using UHF method. Frequency and phase spectrums of EMW signals emitted by PD on different positions were observed. The possibility of application of single-phase PDM device on three-phase GIS for protrusion on the conductor and protrusion on the tank was investigated. The results are summarized as follows.

The most dominant frequency of EMW in three-phase GIS can be estimated from the cutoff frequency formula for single-phase GIS.

The main difference in frequency spectrum between three-phase and single-phase GIS was found to be the shift of $f_{c}$ to lower value for three-phase GIS.

The presence of three conductors can be considered as increase in an equivalent radius of three-phase conductor by a factor of 1.6 for this GIS model.

The phase of PD phase pattern in the phase spectrum depended on the particle position. Application of single-phase PDM device in three-phase GIS gave some erroneous in diagnosis results. The error is caused by error in phase recognition of PD pattern. The change in phase shift $\beta$ setting in PDM device based on the phase angle of applied voltage at maximum electric field on the particle tip gave better diagnosis results.

In actual situation, the particle position is unknown, so that the proper phase shift $\beta$ can not be determined. Therefore, PD data base resulted from different PD types at different positions inside three-phase GIS is needed to achieve correct diagnosis results. More over, modification in PD diagnosis algorithm is needed because three-phase PD data base may be more complicated than single-phase one.

\section{References}

[1] Umar Khayam, S. Ohtsuka, S. Matsumoto, M. Hikita, "Partial Discharge and Cross Interference Phenomena in a Three-phase Construction", International Journal on Electrical Engineering and Informatics, Vol. 1, No.1, pp. 78-91, 2009.

[2] Umar Khayam, T. Ishitobi, S. Ohtsuka, S. Matsumoto, M. Hikita, "Effect of Elliptical Nature of Rotating Electric Field on Partial Discharge Pattern in a Three-phase Construction", IEEJ Transaction on Fundamentals and Materials, Vol. 127, No.9, pp. 524-530, 2007.

[3] S. Okabe, S. Kaneko, M. Yoshimura, H. Muto, C. Nishida, M. Kamei, "Propagation Characteristics of Electromagnetic Waves in Three-Phase-Type Tank from Viewpoint of Partial Discharge Diagnosis on Gas Insulated Switchgear," IEEE Transactions on Dielectrics and Electrical Insulation", Vol. 16, No. 1, pp. 199-205, 2009.

[4] S. Yanabu, H. Okubo, S. Matsumoto: "Metallic Particle Motion in Three-phase $\mathrm{SF}_{6} \mathrm{Gas}$ Insulated Bus", IEEE Transactions on Power Delivery Vol. PWRD-2, No. 1, pp. 1-6, January 1987.

[5] P. C. J. M. van der Wielen: "On-line Detection and Location of Partial Discharges in Medium-Voltage Power Cables", Doctoral Dissertation, T.U. Eindhoven, 2005.

[6] E. Harkink, F. H. Kreuger, P. H. F. Morshuis: "Partial Discharges in Three-core Belted Power Cables", IEEE Transactions on Electrical Insulation, Vol. 24, No. 4, pp. 591-598, 1989.

[7] F. Endo, S. Matsumoto, H. Hama, Hironaka,'Innovation of GIS Insulation Monitoring Techniques and Application to Remote Monitoring System”, 2006 International Council on Large Electric Systems (CIGRE) Session, paper 15-103, Paris, France, August 27 September 12006.

[8] T. Kato, F. Endo, S. Hironaka, "Sensitivity Calibration of UHF Partial Discharge Monitoring System in GIS", IEEJ Transaction on Power and Energy, Vol.122-B, No.11, pp. 1226-1231, 2002. 
[9] Hitachi Engineering and Services Co. Ltd: "Portable Type of PDM Device", 2006.

[10] S. Meijer, E. Gulski, J.J. Smith,"Pattern Analysis of Partial Discharges in SF $_{6}$ GIS", IEEE Trans. on DEI, Vol.5 No. 6, pp. 830-842, 1998.

[11] Umar Khayam, Shinya Ohtsuka, Satoshi Matsumoto, Masayuki Hikita, "Partial Discharge Measurement on Three Phase Construction", Proceeding of IEEE International Conference on Properties and Application of Dielectric Material, ICPADM 2006, Vol. 1, paper K-4, pp.301-304, Bali, INDONESIA, June 26-30, 2006.

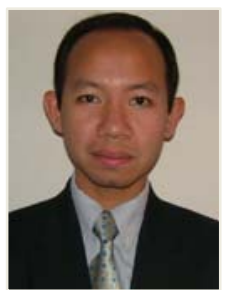

Umar Khayam was born in Indonesia in 1975. He received B.Eng. with honor (cum laude) and M.Eng. degrees in electrical engineering from Bandung Institute of Technology (ITB), Indonesia, in 1998 and 2000, respectively. He received Doctor Degree in Electrical Engineering from Kyushu Institute of Technology(KIT), Japan in 2008. He was a researcher at Hikita Laboratory, KIT, Japan, during 2008-2010. Since 2008, he is a Lecturer at School of Electrical Engineering and Informatics ITB, Indonesia. His research interests include the partial discharge measurement and phenomena in electric power apparatus. Dr. Umar Khayam received Best Paper Award in 2005 KJ Symposium on Electrical Discharge and High Voltage Engineering. He Dr. Umar Khayam can be contacted at School of Electrical Engineering and Informatics ITB, Jl. Ganesha 10 Bandung, Indonesia 40132 or at umar@hv.ee.itb.ac.id.

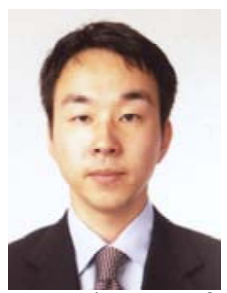

Shinya Ohtsuka was born in 1971. He received his B. S. and M. S. degrees from Kyushu University, Japan, in 1994, 1996, respectively. He finished his Doctor Course of the Deprt. of Electrical and Electronic Systems Engineering at Kyushu University in 1998. He was a research fellow (DC1) of the Japan Society for the Promotion of Science (JSPS) from 1996 to 1998, then became a JSPS Research Fellow (PD) in 1999. Since 1999, he has been an Assistant Professor at Kyushu Institute of Technology, Japan and promoted to Associate Professor at the same university in 2006. His research interests include the insulation properties of environmentally-benign gas as $\mathrm{SF}_{6}$ substitute, $\mathrm{PD}$ detection technique for insulation diagnosis of power apparatus and the superconductivity engineering. He has a Doctor Degree of Engineering. Dr. Ohtsuka is a member of the IEEJ, the IEEDJ and the Cryogenic Society of Japan.

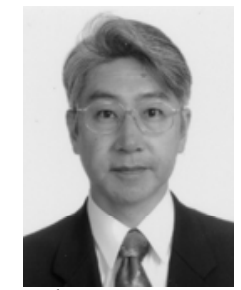

Masayuki Hikita (M'96-SM'00) was born in 1953. He received B. S., M. S. and Dr. degrees in electrical engineering from Nagoya University of Japan, in 1977, 1979, and 1982, respectively. He was an Assistant, a Lecturer, an Associate Professor at Nagoya University in 1982, 1989, and 1992, respectively. Since 1996, he has been a professor of the Department of Electrical Engineering, Kyushu Institute of Technology. He was a Visit Scientist at the High Voltage Laboratory in MIT, USA, from August 1985 to July 1987. Dr. Hikita has recently been interested in research on development of diagnostic technique of electric power equipment. He is a member of the Japan Society of Applied Physics and the IEEJ. He is now a senior member of the IEEE. 
Umar Khayam, et al.

Nobuko Otaka is working at Japan AE Power Systems Corporation, 8-3, Nishi-Shimbashi 3chome, Minato-ku, Tokyo, 105-0003 Japan.

Takakazu Matsuyama is working at Japan AE Power Systems Corporation, 8-3, NishiShimbashi 3-chome, Minato-ku, Tokyo, 105-0003 Japan.

Yoshiki Takehara is working at Hitachi Engineering and Services Co.,Ltd,. 1-1, Kokubo-cho 1-chome, Hitachi-shi, Ibaraki, 316-8501 Japan. 